\title{
Formulações Fortes para o Problema de Dimensionamento de Lotes com Várias Plantas
}

\author{
G.M. MELEGA*, D.J. FIOROTTO e S.A. DE ARAUJO
}

Recebido em 19 dezembro, 2012 / Aceito em 16 abril, 2013

\begin{abstract}
RESUMO. Este trabalho aborda o problema de dimensionamento de lotes em um ambiente com várias plantas. Cada item pode ser produzido em qualquer planta e é possível atender a demanda de uma determinada planta com produção proveniente de uma (ou várias outras) planta(s), para tanto, incorre-se um custo de transferência. O objetivo deste trabalho consiste em desenvolver formulações fortes para o problema. São investigadas reformulações que baseiam-se no problema do caminho mínimo e localização de facilidades. Por fim, alguns resultados computacionais são apresentados comparando todas as formulações propostas.
\end{abstract}

Palavras-chave: dimensionamento de lotes, várias plantas, formulações fortes.

\section{INTRODUÇÃO}

Este artigo trata de problemas de dimensionamento de lotes, o qual é composto de várias plantas integradas com capacidade limitada. Os itens podem ser produzidos em qualquer uma das plantas e para iniciar a produção de um item incorre-se um tempo de preparação na planta utilizada. Cada planta tem uma demanda própria e existe um custo de transferência entre as plantas caso seja necessário transferir para atender a demanda de outra planta. O principal objetivo deste problema é estabelecer a quantidade a ser produzida de cada item em cada uma das plantas em um determinado período.

$\mathrm{Na}$ literatura não existem muitos trabalhos que consideram o ambiente de produção composto com várias plantas. Um dos primeiros estudos que consideram este ambiente de produção é apresentado em [4], em que o objetivo é coordenar os planos de produção e estoque em todas as plantas de modo que o desempenho global e a posição competitiva da empresa seja melhorada. Este problema procura coordenar diferentes funções como o planejamento da produção, estoque, distribuição, dentre outros. São levados em consideração os efeitos da incerteza da demanda

*Autor correspondente: Gislaine Mara Melega

Departamento de Matemática Aplicada, IBILCE, UNESP - Universidade Estadual Paulista, 15054-000 São José do Rio Preto, SP, Brasil. E-mails: gislainemelega@gmail.com; diego_fiorotto@hotmail.com; saraujo@ibilce.unesp.br 
final, dos processos de produção e as restrições de capacidade em cada planta, sendo considerado também a integração entre as áreas de coordenação geral e em cada planta.

Em [18] os autores propõem uma abordagem heurística para resolver o problema com várias plantas, podendo suprir a demanda uma da outra por transferência de produção sendo que, todas as plantas podem produzir os mesmos itens. A fim de remover a violação de capacidade os autores empregam uma rotina de suavização, que consiste de dois módulos: mudança e divisão de lotes.

Logo após, [14] abordam um problema no qual os produtos são manufaturados em uma produção em série. Os autores realizam o estudo deste problema em um ambiente de produção de dois estágios, em que levam em consideração o custo de transporte dos produtos entre estes dois estágios de produção, e a este custo, atribui-se um valor fixo. São apresentados vários algoritmos para o modelo que servem como ferramentas úteis em situações reais.

É tratada em [16] a decisão de dimensionamento de lotes integrada às decisões de transporte de itens entre as plantas de uma mesma indústria, nas quais uma planta produz produtos considerados intermediários e outra processa estes produtos obtendo produtos acabados. O principal objetivo dos autores é compreender as relações gerais existentes nas decisões de mudança de capacidade, alteração dos custos, escolhas de transporte, dentre outros. Os autores apresentam uma formulação do modelo de programação inteira mista e desenvolvem desigualdades válidas empregadas a fim de fortalecer a relaxação linear do modelo.

Em [19] os autores apresentam uma abordagem heurística baseada na relaxação Lagrangiana aplicada às restrições de capacidade a fim de resolver um sistema integrado com várias plantas, vários itens e transferências entre plantas utilizando o modelo apresentado em [18]. Tal heurística foi desenvolvida para resolver um problema real em uma empresa de fabricação de produtos de aço laminado com 4 unidades. Os autores concluíram que, de forma geral, a heurística de aproximação Lagrangiana obtém boas soluções para este problema e para os testes realizados, o número de itens é o fator que causa maior interferência no gap.

Em um trabalho recente, [17] utilizam o modelo proposto em [18] e propõem uma heurística baseada na meta-heurística GRASP (Greedy Randomized Adaptive Search Procedures). Os resultados foram analisados e comparados com os obtidos por [19] sendo que a heurística desenvolvida se mostrou melhor para os problemas com várias plantas. Além disso, a heurística desenvolvida foi competitiva para os problemas com máquinas paralelas, neste caso comparadas com [23].

Um modelo para o problema com várias plantas baseado no problema de localização de facilidades é proposto em [20] em que os autores comparam este com o proposto em [18]. Com os resultados dos testes computacionais os autores concluem que embora o modelo proposto tenha um número de restrições e variáveis maior do que o modelo original, sua relaxação linear assim como os tempos computacionais apresentaram melhores resultados.

É apresentado em [10] um modelo de programação inteira mista para um problema com várias plantas em uma industria de bebidas. Os autores abordam o planejamento a longo prazo das operações que definem o escalonamento e o dimensionamento da produção, cujo objetivo é 
satisfazer a procura estimada minimizando os custos de produção, horas extras e transferência. Os resultados obtidos mostram a interdependência entre o planejamento da produção e a distribuição.

A intenção deste trabalho é estudar e comparar algumas reformulações para este problema. Tais reformulações utilizam redefinições de variáveis e abordagens propostas com sucesso na literatura e utilizadas para reformular outros problemas. O objetivo das reformulações é a melhoria nos limitantes inferiores. Duas das principais reformulações estudadas na literatura e que serão utilizadas no presente trabalho baseiam-se no problema do caminho mínimo (Shortest Path - SP) e no problema de localização de facilidades (Facility Location - FL) apresentadas inicialmente em [7] e [15], respectivamente. Cabe ressaltar a importância de se estudar e obter formulações fortes para estes problemas devido a sua grande complexidade computacional, podendo assim, diminuir consideravelmente os tempos e as árvores de solução dos algoritmos exatos.

Este artigo está organizado da seguinte forma. Na Seção 2, é apresentada a formulação original estudada em [18]. Nesta mesma seção também são propostas mais quatro reformulações para o problema. Na Seção 3, são apresentados os testes computacionais, bem como, são analisadas as comparações realizadas entre todas as formulações. Finalmente, na Seção 4, conclusões e considerações para trabalhos futuros são apresentadas.

\section{FORMULAÇÕES DO PROBLEMA}

Inicialmente será apresentada a formulação original proposta em [18] com a inclusão das variáveis de estoque inicial e em seguida as reformulações propostas para este problema.

Para a formulação matemática do problema considere os seguintes dados:

$I=\{1, \ldots, N\}$ itens, $T=\{1, \ldots, R\}$ períodos e $P=\{1, \ldots, M\}$ plantas;

$s c_{i j t}$ : custo de preparo do item $i$ na planta $j$ no período $t$;

$v c_{i j t}$ : custo de produção do item $i$ na planta $j$ no período $t$;

$h c_{i j t}$ : custo unitário de estoque do item $i$ na planta $j$ no período $t$;

$f c_{i l}$ : custo unitário de estoque inicial para o item $i$ na planta $l$;

$d_{i j t}$ : demanda do item $i$ na planta $j$ durante o período $t$;

$s t_{i j t}$ : tempo de preparo do item $i$ na planta $j$ no período $t$;

$v t_{i j t}$ : tempo de produção do item $i$ na planta $j$ no período $t$;

$r_{j l t}$ : custo unitário de transferência de uma unidade de qualquer item da planta $j$ para a planta $l$ durante o período $t$;

$\mathrm{Cap}_{j t}$ : capacidade (em unidade de tempo) da planta $j$ no período $t$.

\section{Variáveis de decisão:}

$X_{i j t}$ : quantidade produzida do item $i$ na planta $j$ durante o período $t$;

$H_{i j t}$ : quantidade estocada do item $i$ na planta $j$ durante o período $t$;

$H_{i l 0}$ : quantidade de estoque inicial para o item $i$ na planta $l$;

$Z_{i j t}$ : variável binária, indicando a produção ou não do item $i$ na planta $j$ durante o período $t$;

$W_{i j l t}$ : quantidade do item $i$ a ser transferida da planta $j$ para a planta $l(l \neq j)$ durante o período $t$. 
A formulação original para o problema de dimensionamento de lotes com múltiplas plantas (Multi-Plant Capacitated Lot-Sizing Problem-MPCL) é dada por:

$$
\begin{gathered}
\operatorname{Min} \sum_{i=1}^{N} \sum_{l=1}^{M} f c_{i l} H_{i l 0} \\
+\sum_{i=1}^{N} \sum_{j=1}^{M} \sum_{t=1}^{R}\left[s c_{i j t} Z_{i j t}+v c_{i j t} X_{i j t}+h c_{i j t} H_{i j t}+\left(\sum_{\substack{l=1 \\
l \neq j}}^{M} r_{j l t} W_{i j l t}\right)\right]
\end{gathered}
$$

Sujeito a:

$$
\begin{array}{lr}
X_{i j t}+H_{i j t-1}-H_{i j t}+\sum_{\substack{s=1 \\
s \neq j}}^{M} W_{i s j t}-\sum_{\substack{l=1 \\
l \neq j}}^{M} W_{i j l t}=d_{i j t}, & \forall i, \forall j, \forall t . \\
\sum_{i=1}^{N}\left(s t_{i j t} Z_{i j t}+v t_{i j t} X_{i j t}\right) \leq C a p_{j t}, & \forall j, \forall t . \\
X_{i j t} \leq\left(\sum_{j=1}^{M} \sum_{\tau=t}^{R} d_{i j \tau}\right) Z_{i j t}, & \forall i, \forall j, \forall t . \\
Z_{i j t} \in\{0,1\}, & \forall i, \forall j, \forall t . \\
X_{i j t} \geq 0, \quad H_{i l t} \geq 0, \quad H_{i j 0} \geq 0, \quad H_{i j R}=0, \quad W_{i j l t} \geq 0, & \forall i, \forall j, \forall l, j \neq l, \forall t .
\end{array}
$$

A função objetivo (2.1) minimiza $H_{i l 0}$ a soma dos custos de estoque inicial, soma dos custos de preparo, produção, estoque como também os custos de transferência dos itens entre as plantas. As restrições (2.2) garantem o balanceamento de estoque do item $i$ na planta $j$ durante o período $t$, ou seja, a demanda do item $i$ na planta $j$ durante o período $t$ é atendida pela produção deste item na planta $j$ no período $t$, adicionado à quantidade do item armazenada no período anterior na planta $j$, a quantidade a ser transferida de outras plantas para planta $j$, subtraindo a quantidade do item $i$ na planta $j$ no período $t$ que é transferida para as outras plantas e a quantidade deste item que é armazenada em estoque no período $t$ na planta $j$. Em seguida, temos as restrições de capacidade (2.3) garantindo que a capacidade disponível na planta $j$ no período $t$ não é violada. As restrições de preparo (2.4) asseguram que o tempo e o custo de preparo são considerados quando existe produção, isto é, $X_{i j t}>0$ e, por fim, (2.5) e (2.6) são as restrições de domínio das variáveis.

No modelo original proposto em [18], os autores não utilizam nenhum procedimento para evitar possíveis problemas infactíveis. A fim de lidar com este tipo de problema, nos nossos modelos será permitido estoque inicial, em que este, está disponível no primeiro período a um custo alto $\left(f c_{i l}\right)$ de forma que, não é necessário preparo para o estoque inicial. Outras abordagens tem sido utilizadas para tratar a infactibilidade dos problemas, tais como: a permissão de atrasos no atendimento à demanda e a permissão de horas extras ([24]). 


\subsection{Reformulações Baseadas no Problema do Caminho Mínimo}

O problema de dimensionamento de lotes com várias plantas pode ser reformulado usando a abordagem de redefinição das variáveis proposta em [7], originando uma formulação baseada no problema de caminho mínimo. A ideia foi originalmente proposta para problemas sem restrição de capacidade e [13], [12] e [8] estenderam para o caso com restrição de capacidade, máquinas paralelas relacionadas e máquinas paralelas não relacionadas, respectivamente.

Para a reformulação defina os seguintes parâmetros:

- $c v_{i j l t \tau}$ : custo de produção, transferência e estoque total do item $i$, na planta $j$ durante o período $t$, utilizado para satisfazer a demanda do item $i$ na planta $l$ dos períodos $t$ até $\tau$, $\operatorname{com} \tau \geq t, c v_{i j l t \tau}=\left(v c_{i j t}+r_{j l t}\right) \sum_{a=t}^{\tau} d_{i l a}+\sum_{a=t+1}^{\tau} \sum_{b=t}^{a-1} h c_{i l b} d_{i l a} ;$

- $c t_{i j l t \tau}$ : tempo necessário para a produção do item $i$, na planta $j$ durante o período $t$, utilizado para satisfazer a demanda do item $i$ na planta $l$ dos períodos $t$ até $\tau$, $\operatorname{com} \tau \geq t$, $c t_{i j l t \tau}=v t_{i j t} \sum_{a=t}^{\tau} d_{i l a}$;

- $c o_{i l t}$ : custo de estoque inicial para o item $i$ na planta $l$, afim de satisfazer a demanda do período 1 até o período $t, c o_{i l t}=\sum_{a=1}^{t} f c_{i l t} d_{i l a}+\sum_{s=2}^{t} \sum_{u=1}^{s-1} h c_{i l u} d_{i l s}$.

Tem-se também as novas variáveis para o modelo:

- $V_{i j l t \tau}$ : fração do plano de produção do item $i$, na planta $j$ durante o período $t$, utilizado para satisfazer a demanda do item $i$ na planta $l$ dos períodos $t$ até $\tau$, com $\tau \geq t$;

- $V I_{i l t}$ : fração do plano de estoque inicial para o item $i$ na planta $l$, em que a demanda é satisfeita para os primeiros $t$ períodos.

A reformulação baseada no problema do caminho mínimo (Multi-Plant Shortest Path ProblemMPSP) é a seguinte:

$$
\begin{aligned}
\operatorname{Min} & \sum_{i=1}^{N} \sum_{l=1}^{M} \sum_{t=1}^{R} c o_{i l t} V I_{i l t}+\sum_{i=1}^{N} \sum_{j=1}^{M} \sum_{t=1}^{R} s c_{i j t} Z_{i j t} \\
& +\sum_{i=1}^{N} \sum_{j=1}^{M} \sum_{l=1}^{M} \sum_{t=1}^{R} \sum_{\tau=t}^{R} c v_{i j l t \tau} V_{i j l t \tau}
\end{aligned}
$$

Sujeito a:

$$
\begin{array}{lr}
\sum_{\tau=1}^{R} V I_{i l \tau}+\sum_{j=1}^{M} \sum_{\tau=1}^{R} V_{i j l 1 \tau}=1, & \forall i, \forall l . \\
\sum_{j=1}^{M} \sum_{\tau=1}^{t-1} V_{i j l \tau t-1}+V I_{i l t-1}=\sum_{j=1}^{M} \sum_{\tau=t}^{R} V_{i j l t \tau}, & \forall i, \forall l, \forall t, t \geq 2 .
\end{array}
$$




$$
\begin{array}{lr}
\sum_{i=1}^{N} s t_{i j t} Z_{i j t}+\sum_{i=1}^{N} \sum_{l=1}^{M} \sum_{\tau=t}^{R} c t_{i j l t \tau} V_{i j l t \tau} \leq C a p_{j t}, & \forall j, \forall t . \\
\sum_{\tau=t}^{R} V_{i j l t \tau} \leq Z_{i j t}, & \forall i, \forall j, l, \forall t . \\
Z_{i j t} \in\{0,1\}, \quad V I_{i l t} \geq 0, & \forall i, \forall j, \forall l, \forall t . \\
V_{i j l t \tau} \geq 0, & \forall i, \forall j, l, \forall t, \tau, \tau \geq t .
\end{array}
$$

Nesta formulação, a função (2.7) minimiza a soma dos custos de estoque inicial, preparo e custo agregado de produção, transferência e estocagem. As restrições (2.8) e (2.9) definem as restrições de fluxo para o problema de caminho mínimo. Para cada item, um fluxo unitário é enviado na rede, impondo que a demanda de cada item $i$, em cada planta $l$ dos períodos de $t$ até $\tau$ seja satisfeita sem atrasos. As restrições de capacidade (2.10) correspondem às restrições (2.3). Em seguida, as restrições (2.11) asseguram que o tempo e o custo de preparo são considerados quando existe produção. Por fim, as restrições (2.12) e (2.13) são de domínio das variáveis.

Com o intuito de aumentar a eficiência de métodos de solução e reduzir o espaço de soluções (região factível), pode-se inserir e/ou substituir inequações válidas nas formulações, obtendo assim, possíveis melhoras nos resultados. Seguindo esta ideia, uma formulação equivalente para o problema acima pode ser obtida do mesmo modo que em [2] substituindo para cada item e para cada planta as restrições de entrada de fluxo (2.8) e a restrições de balanceamento de fluxo no período $t$ (2.9), pela soma das restrições de balanceamento de fluxo para os primeiros $t$ períodos, ou seja,

$$
\sum_{\tau=t}^{R} V I_{i l \tau}+\sum_{j=1}^{M} \sum_{s=1}^{t} \sum_{\tau=t}^{R} V_{i j l s \tau}=1, \quad \forall i, \forall l, \forall t
$$

Chamaremos a formulação (2.7)-(2.13), com as restrições (2.8) e (2.9) substituídas por (2.14) de reformulação transformada baseada no problema do caminho mínimo (Multi-Plant Shortest Path Transformed Problem-MPSPt).

Observe que a diferença entre as reformulações obtidas antes e após a substituição dessas inequações é de que na reformulação $M P S P$ as restrições de fluxo representam o balanceamento de fluxo em cada nó do grafo. Já com as novas restrições (MPSPt) a análise de entrada e saída de fluxo se dá em cada corte de arcos do grafo.

\subsection{Reformulações Baseadas no Problema de Localização de Facilidades}

O problema de dimensionamento de lotes com várias plantas pode também ser reformulado com base em analogias aos problemas de localização de facilidades, utilizando as variáveis originalmente nele empregadas e obtendo desta forma uma reformulação mais forte quando comparada a formulação original. Esta abordagem foi utilizada pela primeira vez em [15] e vem 
sendo utilizada por vários autores para os problemas de dimensionamento de lotes ([21],[22], [6], [3] e [20]). Em [5] os autores demonstram a equivalência entre as relaxações lineares das reformulações $(S P)$ e $(F L)$, para o problema de dimensionamento de lotes com única máquina e com tempo de preparo. Como foi apontado por alguns autores ([1] e [6]) ao considerar o problema com única máquina, a formulação $(S P)$ se mostra mais rápida computacionalmente e possui $O(R N)$ restrições e $O\left(R^{2} N\right)$ variáveis, enquanto a formulação $(F L)$ apresenta uma matriz de restrições menos densa envolvendo menos coeficientes e possui, $O\left(R^{2} N\right)$ variáveis e restrições, onde $N$ é o número de itens e $R$ é o número de períodos ([5]).

Para obter tal reformulação é necessária a definição dos seguintes parâmetros:

- $c v_{i j l t \tau}$ : custo de produção, transferência e estoque total do item $i$, na planta $j$ durante o período $t$, utilizado para satisfazer a demanda do item $i$ na planta $l$ em um período posterior $\tau, \operatorname{com} \tau \geq t, c v_{i j l t \tau}=\left(v c_{i j t}+r_{j l t}\right) d_{i l \tau}+\sum_{b=t}^{\tau-1} h c_{i l b} d_{i l \tau}$

- $c t_{i j l t \tau}$ : tempo necessário para a produção do item $i$, na planta $j$ durante o período $t$, utilizado para satisfazer a demanda do item $i$ na planta $l$ em um período $\tau$, $\operatorname{com} \tau \geq t$, $c t_{i j l t \tau}=v t_{i j t} d_{i l \tau}$

- $c o_{i l t}$ : custo de estoque inicial para o item $i$ na planta $l$, utilizado para satisfazer a demanda no período $t, c o_{i l t}=f c_{i l t} d_{i l t}+\sum_{b=1}^{t-1} h c_{i l b} d_{i l t}$.

Tem-se também as seguintes novas variáveis para o modelo:

- $F_{i j l t \tau}$ : fração do plano de produção do item $i$, na planta $j$ durante o período $t$, utilizado para satisfazer a demanda do item $i$ na planta $l$ em um período posterior $\tau$, $\operatorname{com} \tau \geq t$;

- $F I_{i l t}$ : fração do plano de estoque inicial para o item $i$ na planta $l$, em que a demanda do período $t$ é satisfeita pelo estoque inicial.

Assim, a reformulação baseada no problema de localização de facilidades (Multi-Plant Facility Location Problem - MPFL)

$$
\begin{aligned}
\operatorname{Min} & \sum_{i=1}^{N} \sum_{l=1}^{M} \sum_{t=1}^{R} c o_{i l t} F I_{i l t}+\sum_{i=1}^{N} \sum_{j=1}^{M} \sum_{t=1}^{R} s c_{i j t} Z_{i j t} \\
& +\sum_{i=1}^{N} \sum_{j=1}^{M} \sum_{l=1}^{M} \sum_{t=1}^{R} \sum_{\tau=t}^{R} c v_{i j l t \tau} F_{i j l t \tau}
\end{aligned}
$$

Sujeito a:

$$
\begin{array}{ll}
F I_{i l t}+\sum_{j=1}^{M} \sum_{\tau=1}^{t} F_{i j l \tau t}=1, & \forall i, \forall l, \forall t . \\
\sum_{i=1}^{N} s t_{i j t} Z_{i j t}+\sum_{i=1}^{N} \sum_{l=1}^{M} \sum_{\tau=t}^{R} c t_{i j l t \tau} F_{i j l t \tau} \leq C a p_{j t}, & \forall j, \forall t .
\end{array}
$$




$$
\begin{array}{lr}
F_{i j l t \tau} \leq Z_{i j t}, & \forall i, \forall j, l, \forall t, \tau \geq t . \\
Z_{i j t} \in\{0,1\}, \quad F I_{i l t} \geq 0, & \forall i, \forall j, \forall l, \forall t . \\
F_{i j l t \tau} \geq 0, & \forall i, \forall j, l, \forall t, \tau \geq t .
\end{array}
$$

Analisando a reformulação (2.15)-(2.20) tem-se que, a função objetivo (2.15) minimiza a soma dos custos de estoque inicial, preparo e custo agregado de produção, transferência e estocagem. As restrições (2.16) asseguram que a produção total para o item $i$ em todas as plantas $j$ do período 1 até $t$, mais o estoque inicial do item $i$ na própria planta, seja igual a demanda deste item, no período $t$ em uma determinada planta $l$. As restrições de capacidade (2.17) correspondem às restrições (2.3) que limitam a capacidade de cada planta em cada período. As restrições (2.18) asseguram que o tempo e o custo de preparo são considerados quando existe produção. Por fim, as restrições (2.19) e (2.20) são de domínio das variáveis.

Uma outra maneira de analisar a formulação (2.15) - (2.20) é fazer uma correspondência ao problema de localização de facilidades em que, a função objetivo (2.15) minimiza a soma dos custos de estoque inicial, custo fixo de instalação da facilidade e o custo agregado de produção, transferência e estocagem na utilização da facilidade. As restrições (2.16) asseguram que para um dado item $i$ e para todos os clientes (par $(l, t)$ - combinação de plantas e períodos) devese escolher locais de instalação de facilidades (par $(j, \tau)$ - combinação de plantas e períodos anteriores a $t$ ), a fim de atender a demanda do cliente (par $(l, t)$ ). As restrições (2.17) limitam a utilização da capacidade de cada facilidade $(j, t)$, em que é considerado um tempo fixo gasto para a instalação da facilidade e o tempo gasto para a produção dos itens. Em seguida, as restrições (2.18) asseguram que para todo par $(j, t)$ de facilidade instalada, é possível atender a demanda de qualquer par $(l, \tau)$ de clientes. E finalmente, as restrições (2.19) e (2.20) são de domínio das variáveis.

Pode-se obter uma nova reformulação substituindo as restrições de preparo (2.18) pelas inequações de precedência (2.21) e (2.22) dadas a seguir, resultando na formulação denominada MPFLp (Multi-Plant Facility Location Problem with Precedence) ([25]).

$$
\begin{array}{lr}
F_{i j l t t} \leq Z_{i j t}, & \forall i, \forall j, l, \forall t, \tau \geq t . \\
F_{i j l t \tau-1} \geq F_{i j l t \tau}, & \forall i, \forall j, l, \forall t, \tau \geq t+1 .
\end{array}
$$

\section{RESULTADOS COMPUTACIONAIS}

As reformulações propostas na seção anterior, foram escritas na sintaxe do AMPL ([9]) e como solver utilizou-se o CPLEX 12.1. ([11]). Todos os testes foram realizados em um computador com processador Intel Corel i7, com 2,93 GHz e com 8,00 GB de memória RAM, sob a plataforma do Windows 7.

Foram testados, utilizando os mesmos exemplares de [17], um total de 480 exemplares que são divididos em 8 tipos diferentes de classes geradas com valores altos e baixos para os custos de preparação (SA ou SB), para tempos de preparação (TA ou TB) e com capacidades normal e 
apertada (CN ou CA). Portanto, a classe CNSBTB, por exemplo, refere-se ao exemplar com capacidade normal, custos de preparação baixos e tempos de preparação baixos. A notação para as demais classes seguem esse mesmo raciocínio. Ainda, são geradas 5 exemplares para cada classe e configuração de número de plantas $(\mathrm{M}=2,4$ e 6$)$ e ítens $(\mathrm{N}=6,12,25$ e 50) em que são considerados sempre 12 períodos, resultando num total de 60 exemplares para cada classe.

Os parâmetros foram gerados em intervalos $[a ; b]$ com distribuição uniforme e denotados por $U[a ; b]$. Tem-se custo de produção $\left(v c_{i j t}\right) \in \mathrm{U}[1,5 ; 2,5]$; custo de preparação $\left(s c_{i j t}\right) \in$ $\mathrm{U}[5,0 ; 95,0]$; custo de estoque $\left(h c_{i j t}\right) \in \mathrm{U}[0,2 ; 0,4]$; custo de transferência $\left(r_{j l t}\right) \in \mathrm{U}[0,2 ; 0,4]$; tempo de produção $\left(v t_{i j t}\right) \in \mathrm{U}[1,0 ; 5,0]$; tempo de preparação $\left(s t_{i j t}\right) \in \mathrm{U}[10,0 ; 50,0]$; demanda $\left(d_{i j t}\right) \in \mathrm{U}[0 ; 180]$; estoque inicial $\left(f c_{i j}\right)=9999$.

Para gerar exemplares com custos de preparação alto multiplicam-se os custos gerados por 10. Da mesma forma, para gerar exemplares com tempos de preparação alto multiplicam-se estes por 1,5 .

A capacidade também foi gerada segundo [17], ou seja, toma-se como base a capacidade necessária para produzir os itens de acordo com a política lote-por-lote. Posteriormente aplica-se um ajuste para reduzir a capacidade a fim de gerar exemplares com a utilização da capacidade em torno de $80 \%$. A capacidade apertada é obtida multiplicando a capacidade gerada por 0,9 . Por fim, para cada exemplar foi considerado um tempo limite de 30 minutos (1800 segundos).

Os resultados computacionais obtidos com a realização dos testes para todas as formulações (MPCL, MPFL, MPFLp, MPSP e MPSPt) serão analisados segundo os seguintes quesitos: relaxação linear, limite superior, limite inferior, gap, tempo, número de nós e número de cortes.

A Tabela 1 apresenta a média geral da relaxação linear $(R L)$ para cada classe de problemas. Foi observado nos resultados que todas as reformulações apresentam os mesmos valores para as relaxações lineares em todas as classes, por isso omitimos a coluna da relaxação linear nas demais reformulações. Tal fato sugere uma extensão da equivalência demonstrada em [5] para o problema com uma única máquina. Além disso, estes valores são sempre melhores quando comparados à formulação original, sendo que esta diferença é de aproximadamente $12 \%$ na média geral. Observe ainda que as maiores diferenças são obtidas no conjunto de classes com custo de preparo alto, em que as reformulações obtém resultados até $16 \%$ melhores.

Para algum exemplares de problemas os resultados obtidos com limitantes inferiores, superiores e gap são inconclusos, ou seja, apresentam valores com diferentes tamanhos decimais. Isto se deve ao fato de que nestas classes, com exceção da formulação MPSPt, as formulações não obtiveram soluções factíveis para algumas exemplares no tempo estipulado sem o uso das variáveis de estoque inicial, que possuem um custo muito elevado. Este fato ocorreu especificamente nas classes com custos de preparo alto e exemplares com 25 itens, 4 e 6 plantas e 50 itens, 4 e 6 plantas. Portanto, para uma análise mais clara, tais exemplares foram desconsiderados para as tabelas com limite inferior $(L I)$, superior $(L S)$ e gap. Ao analisar os limitantes inferiores (ver a Tabela 1), nota-se que de forma geral houve um nivelamento entre a formulação original e as reformulações. 
Tabela 1: Relaxação linear e limite inferior.

\begin{tabular}{|l|cc|cc|c|c|c|}
\hline & \multicolumn{2}{|c|}{ CL/MP } & \multicolumn{2}{c|}{ MPFL } & MPFLp & MPSP & MPSPt \\
\hline & RL & LI & RL & LI & LI & LI & LI \\
\hline CASATA & 221491,24 & 138334,62 & 258156,50 & 140783,56 & 140657,66 & 140582,46 & 140620,12 \\
CASATB & 222378,34 & 139801,72 & 259519,99 & 141804,34 & 142142,09 & 141917,15 & 141909,52 \\
CASBTA & 193210,19 & 205830,88 & 205817,06 & 205955,49 & 205960,39 & 205952,39 & 205963,77 \\
CASBTB & 193617,54 & 206236,60 & 206220,90 & 206362,47 & 206366,04 & 206358,26 & 206369,19 \\
CNSATA & 220004,93 & 136121,14 & 255462,19 & 137833,04 & 137834,53 & 137709,66 & 137736,28 \\
CNSATB & 220543,16 & 136820,33 & 256136,26 & 138509,47 & 138488,96 & 138330,76 & 138376,14 \\
CNSBTA & 192680,18 & 205105,28 & 205111,18 & 205218,73 & 205221,40 & 205220,04 & 205220,12 \\
CNSBTB & 192850,65 & 205281,55 & 205282,10 & 205399,17 & 205399,37 & 205398,68 & 205399,85 \\
MÉDIA & 207097,03 & 171691,52 & 231463,27 & 172733,28 & 172758,81 & 172683,67 & 172699,37 \\
\hline
\end{tabular}

A Tabela 2, apresenta a média para o limite superior $(L S)$ e gap em cada classe de problemas. Os limitantes superiores encontrados pela formulação original são ligeiramente melhores que os das reformulações. Isso se deve ao fato de que nas reformulações o tempo utilizado para resolver a relaxação linear é maior se comparado a formulação original, portanto, analisa-se uma quantidade menor de nós, resultando em um limite superior de pior qualidade. Em relação ao gap, todas as reformulações apresentam na média geral, melhorias, quando comparados a formulação original, pois as diferenças na qualidade entre os limites inferiores (que são melhores nas reformulações) são maiores se comparadas aos limites superiores (que são ligeiramente melhore na formulação original). Destaca-se que a reformulação MPSPt em geral apresenta um desempenho melhor na resolução destes problemas.

Tabela 2: Limite superior e gap.

\begin{tabular}{|l|cc|cc|cc|cc|cc|}
\hline & \multicolumn{3}{|c|}{ MPCL } & \multicolumn{2}{c|}{ MPFL } & \multicolumn{2}{c|}{ MPFLp } & \multicolumn{2}{c|}{ MPSP } & \multicolumn{2}{c|}{ MPSPt } \\
\hline & LS & GAP & LS & GAP & LS & GAP & LS & GAP & LS & GAP \\
\hline CASATA & 141162,26 & 1,98 & 143278,51 & 2,04 & 143196,79 & 2,09 & 142857,89 & 1,99 & 142766,40 & 1,89 \\
CASATB & 142708,00 & 1,95 & 144486,67 & 2,21 & 144987,02 & 2,29 & 144366,67 & 2,08 & 144571,18 & 2,28 \\
CASBTA & 205998,16 & 0,09 & 206069,09 & 0,07 & 206067,14 & 0,07 & 206060,28 & 0,07 & 206056,07 & 0,06 \\
CASBTB & 206434,38 & 0,10 & 206501,59 & 0,08 & 206505,17 & 0,08 & 206491,93 & 0,09 & 206484,43 & 0,08 \\
CNSATA & 138721,00 & 1,57 & 139403,84 & 1,30 & 139419,51 & 1,30 & 139164,62 & 1,27 & 139098,96 & 1,20 \\
CNSATB & 139672,92 & 1,74 & 140465,29 & 1,64 & 140381,60 & 1,53 & 140060,49 & 1,50 & 139911,19 & 1,35 \\
CNSBTA & 205185,40 & 0,04 & 205266,53 & 0,03 & 205266,52 & 0,03 & 205260,42 & 0,03 & 205260,05 & 0,03 \\
CNSBTB & 205378,25 & 0,05 & 205454,21 & 0,03 & 205453,10 & 0,03 & 205447,51 & 0,03 & 205447,12 & 0,03 \\
MÉDIA & 173157,55 & 0,94 & 173865,72 & 0,93 & 173909,61 & 0,93 & 173713,73 & 0,88 & 173699,43 & 0,86 \\
\hline
\end{tabular}

A Tabela 3 apresenta o número total de cortes gerados e o número de nós analisados em cada classe de problemas. Ao analisar os resultados, nota-se que em relação ao número de cortes gerados todas as reformulações geram cerca de $98 \%$ de cortes a menos que a formulação original. 
Tabela 3: Número de nós e número de cortes.

\begin{tabular}{|l|cc|cc|cc|cc|cc|}
\hline & \multicolumn{2}{|c|}{ MPCL } & \multicolumn{2}{c|}{ MPFL } & \multicolumn{2}{c|}{ MPFLp } & \multicolumn{2}{c|}{ MPSP } & \multicolumn{2}{c|}{ MPSPt } \\
\hline & Nós & Cortes & Nós & Cortes & Nós & Cortes & Nós & Cortes & Nós & Cortes \\
\hline CASATA & 64591 & 1973 & 9424 & 26 & 8301 & 22 & 20800 & 24 & 26513 & 26 \\
CASATB & 68198 & 1951 & 10431 & 25 & 9897 & 24 & 23721 & 20 & 33984 & 27 \\
CASBTA & 62831 & 2104 & 31629 & 26 & 28220 & 30 & 44181 & 20 & 63591 & 20 \\
CASBTB & 55465 & 2104 & 26076 & 27 & 25237 & 29 & 34665 & 22 & 55697 & 21 \\
CNSATA & 56478 & 1945 & 8105 & 22 & 7189 & 21 & 17292 & 22 & 21845 & 22 \\
CNSATB & 57569 & 1966 & 8908 & 22 & 7733 & 22 & 19280 & 17 & 29207 & 24 \\
CNSBTA & 57877 & 2062 & 13410 & 22 & 11521 & 27 & 23909 & 18 & 22213 & 17 \\
CNSBTB & 58441 & 2091 & 17991 & 23 & 16243 & 28 & 32129 & 21 & 34284 & 17 \\
MÉDIA & 60181 & 2025 & 15747 & 24 & 14293 & 25 & 26997 & 21 & 33984 & 22 \\
\hline
\end{tabular}

É possível que este fato tenha ocorrido devido as reformulações serem mais fortes, o que reduz a necessidade da aplicação de cortes para a melhoria da relaxação linear. Em relação ao número de nós analisados as diferenças entre as reformulações e a formulação original continuam muito significativas, uma vez que na média, as reformulações analisam aproximadamente $62 \%$ menos nós. Isso se deve ao fato das reformulações terem um número elevado de variáveis contínuas em relação a formulação original, como consequência, a resolução da relaxação linear em cada nó é mais demorada e analisa-se um número menor de nós no tempo estabelecido. Diferentemente dos números totais de cortes gerados, em que todas as reformulações obtém resultados semelhantes, para o número de nós analisados as diferenças são significantes sendo que as reformulações (MPFLp) e (MPSPt) apresentam as maiores diferenças.

Com a Tabela 4 analisa-se as diferenças entre os tempos computacionais em segundos (coluna tempo) entre as reformulações e a formulação original. Para o número de exemplares em que provou-se a otimalidade (coluna SO) as diferenças são ainda maiores, umas vez que nas reformulações propostas prova-se a otimalidade para mais que o dobro dos exemplares, em que mais uma vez a reformulação MPSPt apresenta os melhores resultados.

Tabela 4: Tempo computacional e soluções ótimas.

\begin{tabular}{|l|cc|cc|cc|cc|cc|}
\hline & \multicolumn{2}{|c|}{ CL/MP } & \multicolumn{2}{c|}{ MPFL } & \multicolumn{2}{c|}{ MPFLp } & \multicolumn{2}{c|}{ MPSP } & \multicolumn{2}{c|}{ MPSPt } \\
\hline & Tempo & SO & Tempo & SO & Tempo & SO & Tempo & SO & Tempo & SO \\
\hline CASATA & 1766,21 & 13 & 1652,75 & 41 & 1688,17 & 41 & 1704,91 & 42 & 1703,27 & 47 \\
CASATB & 1773,95 & 10 & 1668,27 & 47 & 1717,96 & 47 & 1740,37 & 40 & 1429,66 & 41 \\
CASBTA & 1499,94 & 24 & 1406,36 & 41 & 1375,19 & 41 & 1532,70 & 43 & 1477,63 & 42 \\
CASBTB & 1509,48 & 21 & 1432,38 & 36 & 1428,07 & 36 & 1553,29 & 45 & 1540,10 & 40 \\
CNSATA & 1685,70 & 12 & 1605,74 & 48 & 1625,79 & 48 & 1619,47 & 38 & 1595,43 & 45 \\
CNSATB & 1721,72 & 13 & 1624,84 & 44 & 1637,25 & 44 & 1670,82 & 40 & 1685,14 & 45 \\
CNSBTA & 1188,19 & 32 & 673,40 & 52 & 671,77 & 52 & 743,93 & 52 & 751,41 & 57 \\
CNSBTB & 1325,17 & 32 & 867,00 & 52 & 853,73 & 52 & 1006,30 & 49 & 939,42 & 49 \\
MÉDIA & 1558,80 & 157 & 1366,34 & 361 & 1374,74 & 361 & 1446,47 & 349 & 1429,66 & 366 \\
\hline
\end{tabular}




\title{
4 CONCLUSÕES
}

Neste trabalho foi estudado um problema de dimensionamento de lotes com várias plantas. O objetivo foi propor novas reformulações baseadas no problema do caminho mínimo e no problema de localização de facilidades e compará-las a formulação original estudada em [18].

Embora reformulações propostas tenham um número de maior de variáveis e restrições, a partir da análise dos resultados computacionais, conclui-se que todas as reformulações propostas obtiveram resultados melhores que a formulação original para praticamente todos as características e exemplares analisados. A se destacar a relaxação linear em que na média mostraram-se $12 \%$ melhores e o número maior de exemplares para os quais as reformulações propostas provaram a otimalidade, comprovando a tese de que as reformulações apresentariam resultados significativamente melhores. Observou-se ainda que apesar de competitivas a reformulação $M P S P_{t}$ mostrou-se ligeiramente melhor que as outras reformulações.

Tendo em vista estender o trabalho, tem-se como próximos passos a realização de testes computacionais com outros exemplares da literatura e se necessário o desenvolvimento de uma heurística. Outro tema interessante a ser explorado em pesquisa futura é o estudo de modelos que consideram a economia de escala.

\section{AGRADECIMENTOS}

Os autores agradecem a Mariá C.V. Nascimento e Franklina M.B. Toledo pelo fornecimento dos exemplares utilizados neste trabalho. Agradecem também aos revisores da revista TEMA cujos comentários contribuíram para a qualidade da escrita, discussão e apresentação deste trabalho. Esta pesquisa teve o apoio financeiro da Fundação de Amparo à Pesquisa do Estado de São Paulo (FAPESP - processos: 2010/12796-6, 2010/16727-9 e 2011/22647-0) e Conselho Nacional de Desenvolvimento Científico e Tecnológico (CNPq).

\begin{abstract}
This paper considers the multi-plant lot sizing problem. Each item can be produced in any plant and it is possible to meet the demand of a particular plant with production from one (or several) other plants, in this case, incurs a transfer cost. The objective is to develop strong formulations for this problem. Reformulations that based on the shortest path problem and facility location problem are investigated. Finally, some computational results are presented comparing all the proposed formulations.
\end{abstract}

Keywords: lot sizing, multi-plant, strong formulations.

\section{REFERÊNCIAS}

[1] A. Alfieri, P. Brandimarte \& S. D'orazio. Lp-based heuristics for the capacitated lot-sizing problem: The interaction of model formulation and solution algorithm. International Journal of Production Research, 40 (2002), 441-458. 
[2] S.A. de Araujo, B. de Reyck, Z. Degraeve, I. Fragkos \& R. Jans. Primal and dual bounds for longhorizon lot size problems with tight capacity constraints, em "International Workshop on Lot Sizing - IWLS”, Istanbul-Turquia, (2011).

[3] E.D. Bernardes, S.A. Araujo \& M.S.N. Rangel. Reformulação para um problema integrado de dimensionamento e sequenciamento de lotes. Pesquisa Operacional, 30 (2010), 637-655.

[4] R. Bhatnagar, P. Chandra \& S.K. Goyal. Models for multi-plant coordination. European Journal of Operational Research, 67 (1993), 141-160.

[5] M. Denizel, F. Altekin, H. Sural \& H. Stadtler. Equivalence of the LP relaxations of two strong formulations for the capacitated lot-sizing problem with setup times. OR Spectrum, 30 (2008), $773-$ 785.

[6] M. Denizel \& H. Sural. On alternative mixed integer programming formulations and lp-based heuristics for lot-sizing with setup times. Journal of the Operational Research Society, 57 (2006), 389-399.

[7] G.D. Eppen \& R.K. Martin. Solving multi-item capacitated lot-sizing problems using variable redefinition. Operations Research, 35 (1987), 832-848.

[8] D. J. Fiorotto \& S.A. de Araujo. Relaxação lagrangiana aplicada ao problema de dimensionamento de lotes em máquinas paralelas: Limitantes inferiores. TEMA Tendências em Matemática Aplicada e Computacional, 13 (2012), 13-24.

[9] R. Fourer, D.M. Gay \& B.W. Kernighan. "AMPL: A Modeling Language for Mathematical Programming”, Duxbury Press, Cole Publishing Company, (2002).

[10] L. Guimarães, D. Klabjan \& B. Almada-Lobo. Annual production budget in the beverage industry. Engineering Applications of Artificial Intelligence, 25 (2012), 229-241.

[11] IBM (2007). ILOG - CPLEX 12,1 - Matematic Programing Optimizers.

[12] R. Jans. Solving Lot-Sizing Problems on Parallel Identical Machines Using Symmetry-Breaking Constraints. INFORMS Journal on Computing, 21 (2009), 123-136.

[13] R. Jans \& Z. Degraeve. Improved lower bounds for the capacitated lot sizing problem with setup times. Operations Research Letters, 32 (2004), 185-195.

[14] P. Kaminsky \& D. Simchi-Levi. Production and distribution lot sizing in a two stage supply chain. IIE Transactions, 35 (2003), 1065-1075.

[15] J. Krarup \& O. Bilde. Sharp lower bounds and efficient algorithms for the simple plant location problem, em "Annals of Discrete Mathematics", (1977).

[16] R. Matta \& T. Miller. Production and inter-facility transportation scheduling for a process industry. European Journal of Operational Research, 158 (2004), 72-88.

[17] M.C. Nascimento, M.G. Resende \& F.M. Toledo. Grasp heuristic with path-relinking for the multiplant capacitated lot sizing problem. European Journal of Operational Research, 200 (2010), 747 754.

[18] M. Sambasivan \& C.P. Schmidt. A heuristic procedure for solving multi-plant, multi-item, multiperiod capacitated lot-sizing problems. Asia - Pacific Journal of Operational Research, 19 (2002), 87-105. 
[19] M. Sambasivan \& S. Yahya. A lagrangean-based heuristic for multi-plant, multi-item, multi-period capacitated lot-sizing problems with inter-plant transfers. Computers \& Operations Research, 32 (2005), 537-555.

[20] D.H. Silva \& F.M. Toledo. Dimensionamento de lotes com múltiplas plantas: comparação entre dois modelos, em "Anais do CLAIO/SBPO", (2012).

[21] H. Stadtler. Mixed integer programming model formulations for dynamic multi-item multi-level capacitated lotsizing. European Journal of Operational Research, 94 (1996), 561-581.

[22] C. Suerie \& H. Stadtler. The capacitated lot-sizing problem with linked lot sizes. Management Science, 49 (2002), 1039-1054.

[23] F.M.B. Toledo \& V.A. Armentano. A Lagrange-based heuristic for the capacitated lot-sizing problem with parallel machines. European Journal of Operational Research, 175 (2006), 1070-1083.

[24] F. Vanderbeck. Lot-sizing with start-up times. Management Science, 44 (1988), 1409-1425.

[25] L. Wolsey. Uncapacitated lot-sizing problems with start-up costs. Operational Research, 37 (1989), 741-747. 